\title{
Patterns of aerosol over Malaysia from multiple satellite-borne sensors
}

\begin{abstract}
Aerosols patterns over Malaysia are examined using two different satellite-borne sensors, namely the Moderate Resolution Imaging Spectroradiometer (MODIS) and the Ozone Monitoring Instrument (OMI), which have been providing continuous aerosol data since July 2002 and November 2004 respectively. The spatial and temporal distributions of aerosols detected by MODIS and OMI are described and compared with ground-based particulate matter measurements data. Data were analysed and discussed within the January 2005 until December 2008 time period. This period was chosen so as to compare multiple sources. Validation of MODIS and OMI with ground data for 48 months demonstrated that MODIS data product was more accurate in representing aerosols near the ground, while OMI data product was more accurate in detecting extreme emission events. An assessment of seasonal variability of MODIS, OMI and ground data revealed similar seasonal variability, where maximum values during the southwest monsoon season were found over all stations involved in this study. Therefore, these results suggest MODIS and OMI data product can be used as a proxy for regional aerosol studies.
\end{abstract}

Keyword: Aerosols; Remote sensing; MODIS; OMI 